Centre de Recherches Mathématiques CRM Proceedings and Lecture Notes

Volume 00, 0000

\title{
Derivation and Justification of Plate Models by Variational Methods
}

\author{
Stephen M. Alessandrini, Douglas N. Arnold, \\ Richard S. Falk, and Alexandre L. Madureira
}

\begin{abstract}
We consider the derivation of two-dimensional models for the bending and stretching of a thin three-dimensional linearly elastic plate using variational methods. Specifically we consider restriction of the trial space in two different forms of the Hellinger-Reissner variational principle for 3-D elasticity to functions with a specified polynomial dependence in the transverse direction. Using this approach many different plate models are possible and we classify and investigate the most important. We study in detail a method which leads naturally not only to familiar plate models, but also to error bounds between the plate solution and the full 3-D solution.
\end{abstract}

\section{Introduction}

Let $\Omega$ be a smoothly bounded domain in $\mathbb{R}^{2}$ and $t \in(0,1]$. We consider an isotropic, homogeneous, linearly elastic plate occupying the region $P_{t}=\Omega \times$ $(-t / 2, t / 2)$. Denote the union of the top and bottom surfaces of the plate by $\partial P_{t}^{ \pm}=\Omega \times\{-t / 2, t / 2\}$ and the lateral boundary by $\partial P_{t}^{\mathrm{L}}=\partial \Omega \times(-t / 2, t / 2)$. See Figure 1. We suppose that the plate is loaded by a surface force density $\underline{g}: \partial P_{t}^{ \pm} \rightarrow \mathbb{R}^{3}$ and a volume force density $\underline{f}: P_{t} \rightarrow \mathbb{R}^{3}$, and is clamped along its lateral boundary. The resulting stress $\underline{\underline{\sigma}}^{*}: P_{t} \rightarrow \mathbb{R}_{\mathrm{sym}}^{3 \times 3}$ and displacement $\underline{u}^{*}: P_{t} \rightarrow \mathbb{R}^{3}$ then satisfy the boundary-value problem

$$
\begin{gathered}
\underline{\underline{\underline{\underline{A}}}} \underline{\underline{\sigma}}^{*}=\underline{\underline{\varepsilon}}\left(\underline{u}^{*}\right), \quad-\operatorname{div} \underline{\underline{\sigma}}^{*}=\underline{f} \text { in } P_{t}, \\
\underline{\underline{\sigma}}^{*} \underline{n}=\underline{g} \text { on } \partial P_{t}^{ \pm}, \quad \underline{u}^{*}=0 \text { on } \partial P_{t}^{\mathrm{L}} .
\end{gathered}
$$

Here $\underline{\underline{\varepsilon}}\left(\underline{u}^{*}\right)$ denotes the infinitesimal strain tensor associated to the displacement vector $\underline{u}^{*}$, namely the symmetric part of its gradient, and $\underline{\operatorname{div}} \underline{\underline{\sigma}}$ denotes the vector

1991 Mathematics Subject Classification. Primary: 73K10; Secondary: 73C02.

The second author was supported NSF grant DMS-9500672 and by the Institute for Mathematics and its Applications. The third author was supported by NSF grant DMS-9403552. The fourth author was supported by a fellowship from CNPq-Brazil.

This is the final form of the paper. 


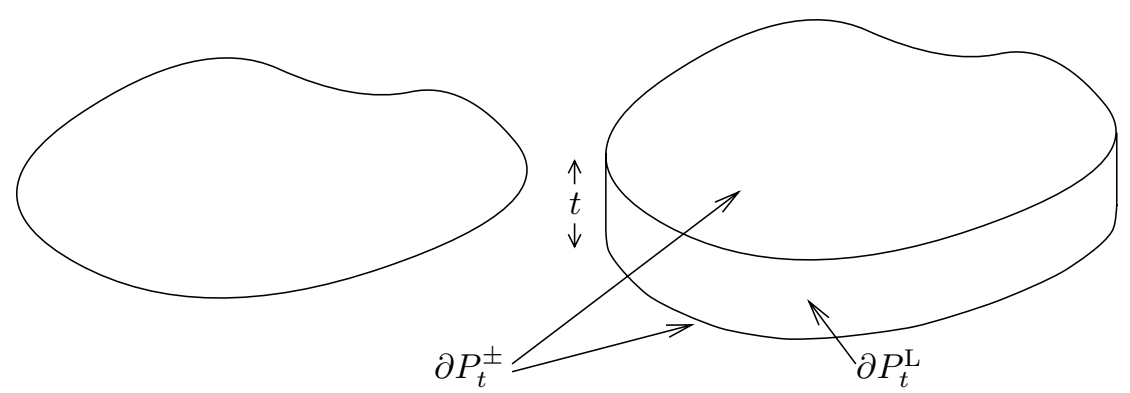

Figure 1. The two-dimensional domain $\Omega$ and plate domain $P_{t}$.

divergence of the symmetric matrix $\underline{\underline{\sigma}}$ taken by rows. The compliance tensor $\underline{\underline{\underline{A}}}$ is given by $\underline{\underline{\underline{A}}} \underline{\underline{\tau}}=(1+\nu) \underline{\underline{\tau}} / E-\nu \operatorname{tr}(\underline{\underline{\tau}}) \underline{\underline{\delta}} / E$, with $E>0$ Young's modulus, $\nu \in[0, \underline{\overline{1}} / 2)$ Poisson's ratio, and $\underline{\underline{\delta}}$ the $3 \times 3$ identity matrix.

A plate model seeks to approximate the solution of the elasticity problem (1) in terms of the solution of a system of partial differential equations on the twodimensional domain $\Omega$ without requiring the solution of a three-dimensional problem. The passage from the $3-\mathrm{D}$ problem to a plate model is known as dimensional reduction.

In this paper we discuss systematic procedures of dimensional reduction to plate models based on variational formulations of the three-dimensional problem (1). In addition to the derivation of plate models, we are very concerned with their rigorous justification in the sense of proving convergence and rates of convergence, in an appropriate sense, of the plate model solution to the 3-D solution as the plate thickness tends to zero.

Before proceeding, we introduce some notation. As has already been seen, we indicate tensors in three variables with underbars. A first-order tensor (or 3vector) is written with one underbar, a second-order tensor (or $3 \times 3$ matrix) with two underbars, etc. For tensors in two variables we use undertildes in the same way. By way of illustration, any 3-vector may be expressed in terms of a 2-vector giving its in-plane components and a scalar giving its transverse component, and any $3 \times 3$ symmetric matrix may be expressed in terms of a $2 \times 2$ symmetric matrix, a 2-vector, and a scalar thus:

$$
\underline{v}=\left(\begin{array}{c}
v \\
\underset{v_{3}}{v}
\end{array}\right), \quad \underline{\underline{\tau}}=\left(\begin{array}{cc}
\underset{\sim}{\tau} & \underset{\sim}{\tau} \\
\underset{\sim}{\tau^{T}} & \tau_{33}
\end{array}\right) .
$$

Underbars and undertildes will be used for tensor-valued functions, operators yielding such functions, and spaces of such operators, as well. Even without explicit mention, all second-order tensors arising in this paper will be assumed symmetric. Thus, for example, the notation $\underset{\approx}{H^{s}}(\Omega)$ denotes the Sobolev space of order $s$ which consists of all functions on $\Omega$ with values in $\mathbb{R}_{\text {sym }}^{2 \times 2}$ whose partial derivatives of order at most $s$ are square integrable. We denote the norm on a (scalar or tensor-valued) Sobolev space of order $s$ on a set $Q$ by $\|\cdot\|_{s, Q}$. In the case where $Q=\Omega$ we just write $\|\cdot\|_{s}$.

By taking odd and even parts with respect to the variable $x_{3}$, the threedimensional plate problem splits into two decoupled problems which correspond 
to stretching and bending of the plate. More precisely, for a function $k(\underline{x})$ defined on $P_{t}$ or $\partial P_{t}^{ \pm}$, define its odd and even parts with respect to $x_{3}$ :

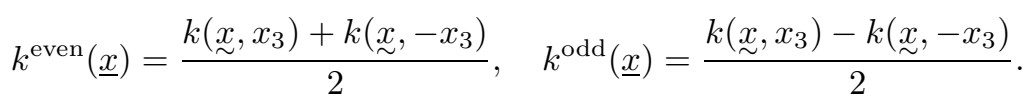

We then decompose the loads

$$
\underline{g}=\underline{g}^{\mathrm{s}}+\underline{g}^{\mathrm{b}}, \quad \underline{f}=\underline{f}^{\mathrm{s}}+\underline{f}^{\mathrm{b}},
$$

with

$$
\underline{g}^{\mathrm{s}}=\left(\begin{array}{c}
\underline{g}^{\text {even }} \\
\tilde{g}_{3}^{\text {odd }}
\end{array}\right), \quad \underline{g}^{\mathrm{b}}=\left(\begin{array}{c}
g^{\text {odd }} \\
\widetilde{g}_{3}^{\text {even }}
\end{array}\right), \quad \underline{f}^{\mathrm{s}}=\left(\begin{array}{c}
f^{\text {even }} \\
\tilde{f}_{3}^{\text {odd }}
\end{array}\right), \quad \underline{f}^{\mathrm{b}}=\left(\begin{array}{c}
f^{\text {odd }} \\
\widetilde{f}_{3}^{\text {even }}
\end{array}\right)
$$

Define the stretching portion $\left(\underline{\underline{\sigma}}^{* \mathrm{~s}}, \underline{u}^{* \mathrm{~s}}\right)$ of the solution by the system (1) with $\underline{g}$ replaced by $g^{\mathrm{s}}$ and $f$ replaced by $f^{\mathrm{s}}$, and define the bending portion of the solution analogously. It is then easy to see that

$$
\begin{aligned}
& \underline{u}^{* \mathrm{~s}}=\left(\begin{array}{c}
u^{* \text { even }} \\
u_{3}^{* \text { odd }}
\end{array}\right), \quad \underline{\underline{\sigma}}^{* \mathrm{~s}}=\left(\begin{array}{cc}
\underset{\sim}{\sigma^{* \text { even }}} & \sigma^{\text {*odd }} \\
\left({\underset{\sim}{\sigma}}^{\text {*odd }}\right)^{T} & \sigma_{33}^{* \text { even }}
\end{array}\right), \\
& \underline{u}^{* \mathrm{~b}}=\left(\begin{array}{c}
\underset{u^{* \text { odd }}}{u_{3}^{* \text { even }}}
\end{array}\right), \quad \underline{\underline{\sigma}}^{* \mathrm{~b}}=\left(\begin{array}{cc}
\underset{\sim}{\sigma^{* \text { odd }}} & \sigma^{* \text { even }} \\
\left({\underset{\sim}{\sigma}}^{* \text { even }}\right)^{T} & \sigma_{33}^{* \text { odd }}
\end{array}\right),
\end{aligned}
$$

and that

$$
\underline{u}=\underline{u}^{\mathrm{s}}+\underline{u}^{\mathrm{b}}, \quad \underline{\underline{\sigma}}=\underline{\underline{\sigma}}^{\mathrm{s}}+\underline{\underline{\sigma}}^{\mathrm{b}} .
$$

Thus, the three-dimensional elasticity problem (1) splits into two decoupled problems, one for the stretching portion of the solution and one for the bending portion.

All the common two-dimensional plate models admit a similar splitting. The most common plate stretching models are variants of the equations of generalized plane stress. The most common plate bending models are variants of the KirchhoffLove biharmonic plate model or of the Reissner-Mindlin plate model. We speak of variants here, because the specification of the forcing functions for the 2-D differential equations in terms of the 3-D loads $g$ and $f$ differs for different models to be found in the literature, as does the specification of the approximate 3-D stresses and displacements in terms of the solutions of the 2-D boundary-value problems. Moreover, there is a coefficient in the Reissner-Mindlin model, the so-called shear correction factor, which is given different values in the literature. So there is no universally accepted basic two-dimensional model of plate stretching or bending.

\section{A Variational Approach to Dimensional Reduction}

The Hellinger-Reissner principle, or HR for short, gives a variational characterization of the solution to the three-dimensional problem (1). To state this principle we define

$$
\underline{\underline{\Sigma}} \underline{\underline{L}}^{2}\left(P_{t}\right), \quad \underline{V}^{\bullet}=\left\{\underline{v} \in \underline{H}^{1}\left(P_{t}\right): \underline{v}=0 \text { on } \partial P_{t}^{\mathrm{L}}\right\} .
$$

Then HR characterizes $\left(\underline{\underline{\sigma}}^{*}, \underline{u}^{*}\right)$ as the unique critical point (namely a saddle point) of the HR functional

$$
J(\underline{\underline{\tau}}, \underline{v})=\frac{1}{2} \int_{P_{t}} \underline{\underline{\underline{A}}} \underline{\underline{\tau}}: \underline{\underline{\tau}} d \underline{x}-\int_{P_{t}} \underline{\underline{\tau}}: \underline{\underline{\varepsilon}}(\underline{v}) d \underline{x}+\int_{P_{t}} \underline{f} \cdot \underline{v} d \underline{x}+\int_{\partial P_{t}^{ \pm}} \underline{g} \cdot \underline{v} d \underline{x}
$$


TABLE 1. Summary of the principle plate models based on the Hellinger-Reissner principle.

\begin{tabular}{cccccc}
\hline model & $\operatorname{deg}_{3} \underset{\sim}{\sigma}$ & $\operatorname{deg}_{3} \underset{\sim}{\sigma}$ & $\operatorname{deg}_{3} \sigma_{33}$ & $\operatorname{deg}_{3} \underset{\sim}{\sim}$ & $\operatorname{deg}_{3} u_{3}$ \\
\hline $\operatorname{HR}_{1}(p)$ & $p$ & $p-1$ & $p-2$ & $p$ & $p-1$ \\
$\operatorname{HR}_{2}(p)$ & $p$ & $p-1$ & $p$ & $p$ & $p-1$ \\
$\operatorname{HR}_{3}(p)$ & $p$ & $p+1$ & $p$ & $p$ & $p+1$ \\
\hline
\end{tabular}

on $\underline{\underline{\Sigma^{\bullet}}} \times \underline{V}^{\bullet}$. Equivalently, $\left(\underline{\underline{\sigma}}^{*}, \underline{u}^{*}\right)$ is the unique element of $\underline{\underline{\Sigma^{\bullet}}} \times \underline{V}^{\bullet}$ satisfying the weak equations

$$
\begin{gathered}
\int_{P_{t}} \underline{\underline{\underline{A}}}_{\underline{\underline{\sigma}}}^{*}: \underline{\underline{\tau}} d \underline{x}-\int_{P_{t}} \underline{\underline{\varepsilon}}(\underline{u}): \underline{\underline{\tau}} d \underline{x}=0 \quad \text { for all } \underline{\underline{\tau}} \in \underline{\underline{\Sigma}} \\
\int_{P_{t}} \underline{\underline{\sigma}}: \underline{\underline{\varepsilon}}(\underline{v}) d \underline{x}=\int_{P_{t}} \underline{f} \cdot \underline{v} d \underline{x}+\int_{\partial P_{t}^{ \pm}} \underline{g} \cdot \underline{v} d \underline{x} \quad \text { for all } \underline{v} \in \underline{V}^{\bullet} .
\end{gathered}
$$

Plate models may be derived by replacing $\sum^{\bullet}$ and $\underline{V}^{\bullet}$ in HR with subspaces $\cong$ and $\underline{V}$ which admit only a specified polynomial dependence on $x_{3}$ and then defining $(\underline{\underline{\sigma}}, \underline{u})$ as the unique critical point of $J$ over $\underline{\underline{\sum}} \times \underline{V}$. This is equivalent to restricting the trial and test spaces in the weak formulation to $\underline{\underline{\Sigma}} \times \underline{V}$. Now if the subspaces $\underline{\equiv}$ and $\underline{V}$ are chosen carelessly, there may not exist any such critical point or it may not be unique. We can, and shall, insure a unique solution by insisting that $\underline{\underline{\varepsilon}}(\underline{V}) \subset \underline{\underline{\Sigma}}$. To describe the spaces we consider, we write $\operatorname{deg}_{3} v=p$, with $v \in L^{2}\left(P_{t}\right)$ and $p \in \mathbb{Z}$, to mean that $v$ is a polynomial in $x_{3}$ of degree $p$ with coefficients in $L^{2}(\Omega)$. If $p<0$, we take this to mean that $v \equiv 0$.

To describe one family of plate models derived in this way, fix a positive integer $p$, and let

$$
\begin{gathered}
\stackrel{\Sigma}{\underline{\Sigma}}=\left\{\underline{\underline{\tau}} \in \underline{\underline{\Sigma}}^{\bullet}: \operatorname{deg}_{3} \underset{\approx}{\tau} \leq p, \operatorname{deg}_{3} \tau \leq p-1, \operatorname{deg}_{3} \tau_{33} \leq p-2\right\}, \\
\underline{V}=\left\{\underline{v} \in \underline{V}^{\bullet}: \operatorname{deg}_{3} \underset{\sim}{\sim} \leq p, \operatorname{deg}_{3} v_{3} \leq p-1\right\} .
\end{gathered}
$$

We refer to this plate model as the $\operatorname{HR}_{1}(p)$ model.

This family of models and two others, called $\operatorname{HR}_{2}(p)$ and $\operatorname{HR}_{3}(p)$, are specified in Table 1. The most interesting plate models based on HR seem to be those in Table 1 and those which consist of one method from Table 1 applied to the stretching problem and another applied to the bending problem. Thus, for example, with $p$ odd, we may apply $\mathrm{HR}_{3}(p-1)$ to the stretching problem and $\mathrm{HR}_{2}(p)$ to the bending problem. This corresponds to seeking a critical point of the Hellinger-Reissner functional over fields with all components of degree at most $p$.

Each of these choices of space fulfils the condition $\underline{\underline{\varepsilon}}(\underline{V}) \subset \underline{\underline{\Sigma}}$, and so defines a unique $\underline{\underline{\sigma}}$ and $\underline{u}$. It is not hard to see that the coefficients of $\underline{\underline{\sigma}}$ and $\underline{u}$ (as polynomials in $x_{3}$ ) are determined by boundary-value problems posed on $\Omega$ (we shall illustrate a specific example below).

The $\mathrm{HR}_{2}(p)$ and $\mathrm{HR}_{3}(p)$ models satisfy the condition $\underline{\underline{A}}^{-1} \underline{\underline{\varepsilon}}(\underline{V}) \subset \underline{\underline{\Sigma}}$ as well.

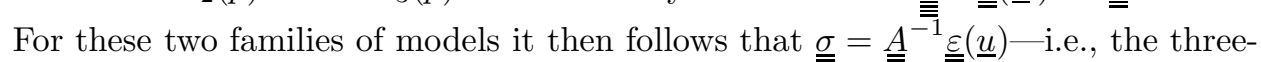
dimensional constitutive equation is satisfied exactly -and that $\underline{u}$ is determined as 
the minimizer in $\underline{V}$ of the potential energy

$$
E(\underline{v})=\frac{1}{2} \int_{P_{t}} \underline{\underline{\underline{\underline{A}}}}^{-1} \underline{\underline{\varepsilon}}(\underline{v}): \underline{\underline{\varepsilon}}(\underline{v}) d \underline{x}-\int_{P_{t}} \underline{f} \cdot \underline{v} d \underline{x}-\int_{\partial P_{t}^{ \pm}} \underline{g} \cdot \underline{v} d \underline{\sim} .
$$

Thus the $\mathrm{HR}_{2}(p)$ and $\mathrm{HR}_{3}(p)$ models are minimum energy models (or projection models), which have been discussed by many authors, for example $[\mathbf{1}, \mathbf{4}, \mathbf{1 2}]$. The $\mathrm{HR}_{1}(p)$ models are not minimum energy models.

We now consider some specific low-order models. For this we introduce notations for the odd and even parts of $\underset{\sim}{g}$ and $g_{3}$ and for the first two moments of $\underset{\sim}{f}$ and $f_{3}$ with respect to $x_{3}$, all viewed as functions on $\Omega$. We set

$$
\begin{gathered}
g_{3}^{0}(\underset{\sim}{x})=\frac{1}{2}\left[g_{3}(\underset{\sim}{x}, t / 2)+g_{3}(\underset{\sim}{x},-t / 2)\right], \quad g_{3}^{1}(\underset{\sim}{x})=\frac{1}{2}\left[g_{3}(\underset{\sim}{x}, t / 2)-g_{3}(\underset{\sim}{x},-t / 2)\right], \\
f_{3}^{0}(\underset{\sim}{x})=\int_{-t / 2}^{t / 2} f_{3}\left(\underset{\sim}{x}, x_{3}\right) d x_{3}, \quad f_{3}^{1}(\underset{\sim}{x})=\int_{-t / 2}^{t / 2} f_{3}\left(\underset{\sim}{x}, x_{3}\right) \frac{x_{3}}{t} d x_{3},
\end{gathered}
$$

and define $\underset{\sim}{g^{0}}, \underset{\sim}{g^{1}}, \underset{\sim}{f}$, and $\underset{\sim}{f}$ analogously.

The $\operatorname{HR}_{1}(\mathbf{1})$ method. Define the two-dimensional analogue of the compliance tensor by $\underset{\approx \sim}{A \tau}=(1+\nu) \underset{\approx}{\tau} / E-\nu \operatorname{tr}(\underset{\approx}{\tau}) \underset{\approx}{\delta} / E$. It can be shown that the $\operatorname{HR}_{1}(1)$ solution is given by

$$
\begin{aligned}
& \underline{u}(\underline{x})=\left(\begin{array}{c}
\underset{\sim}{\eta(\underset{\sim}{x})} \\
0
\end{array}\right)+\left(\begin{array}{c}
\underset{\sim}{-\underset{\omega}{(x)}(\underset{\sim}{x})} x_{3} \\
\underset{\sim}{x})
\end{array}\right)
\end{aligned}
$$

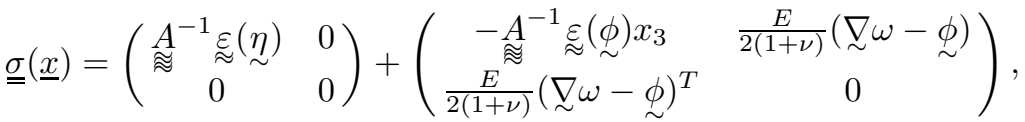

where $\underset{\sim}{\eta}$ is determined by a classical generalized plane stress problem and $\underset{\sim}{\phi}$ and $\omega$ by a Reissner-Mindlin problem. Specifically,

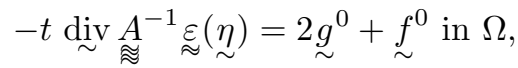

$$
\begin{aligned}
& \underset{\sim}{\sim}=0 \text { on } \partial \Omega,
\end{aligned}
$$

and

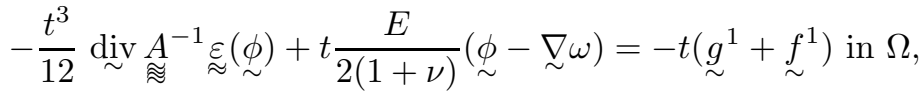

$$
\begin{aligned}
& t \frac{E}{2(1+\nu)} \operatorname{div}(\underset{\sim}{\phi}-\underset{\sim}{\nabla} \omega)=2 g_{3}^{0}+f_{3}^{0} \text { in } \Omega, \\
& \underset{\sim}{\phi}=0, \quad \omega=0 \text { on } \partial \Omega .
\end{aligned}
$$

The verification of these equations is straightforward, but tedious. A similar, but more involved, computation (that for the $\mathrm{HR}_{4}^{\prime}(1)$ model defined in $\S 3$ ) will be given in the appendix.

Note that in the case of a purely transverse bending load, the system (6)-(8) is the classical Reissner-Mindlin system with shear correction factor 1 . When the 
bending is also affected by nonzero $g^{1}$ or $f^{1}$, then these appear as an applied couple in the Reissner-Mindlin system.

Thus we see that the $\operatorname{HR}_{1}(1)$ method is a simple approach to deriving the classical generalized plane stress and Reissner-Mindlin models.

The $\mathrm{HR}_{2}(\mathbf{1})$ model. The conclusion is altogether different for the $\mathrm{HR}_{2}(1)$ model, the simplest minimum energy model. In this case it can be shown that $\underline{u}$ is again given by (2) (and $\underline{\underline{\sigma}}$ is given by an expression a bit more complicated than $(3$ ) which we don't report here), except that the equations (4) and (6) are replaced by

$$
-t[\operatorname{div} \underset{\sim}{\underset{\approx}{A}} \underset{\approx}{\varepsilon}(\underset{\sim}{\eta})+c \underset{\sim}{\nabla} \operatorname{div} \underset{\sim}{\eta}]=2{\underset{\sim}{g}}^{0}+{\underset{\sim}{f}}^{0} \text { in } \Omega
$$

and

$$
-\frac{t^{3}}{12}\left[\operatorname{div}_{\sim} \underset{\approx}{A^{-1}} \underset{\sim}{\underset{\sim}{(\phi)}}(\underset{\sim}{)}+c \underset{\sim}{\nabla} \operatorname{div} \underset{\sim}{\phi}]+t \frac{E}{2(1+\nu)}(\underset{\sim}{\phi}-\underset{\sim}{\nabla} \omega)=-t\left(\underset{\sim}{g}{ }^{1}+f_{\sim}^{1}\right) \text { in } \Omega,\right.
$$

respectively, with $c=-E \nu^{2} /\left[\left(1-\nu^{2}\right)(2 \nu-1)\right]$. These additional terms are spurious, and cause the $\mathrm{HR}_{2}(1)$ model to be divergent as $t$ tends to 0 (in a sense which will be made precise in $\S 4)$.

Thus the $\mathrm{HR}_{2}(1)$ model is incorrect. For $p \geq 3$ it can be shown that the $\mathrm{HR}_{2}(p)$ model is convergent. For $p=3$, it can be shown to be identical to a method of Lo, Christensen, and $\mathrm{Wu}[\mathbf{1 3}]$. However, we feel it possible that even for larger $p$, the $\operatorname{HR}_{2}(p)$ method is both more complicated than and less accurate than the $\operatorname{HR}_{1}(p)$ method.

The $\mathrm{HR}_{\mathbf{3}}(\mathbf{1})$ model. For lack of space we will not discuss this model here, except to note that it is a convergent model, but that the stretching system it yields is more complicated than the generalized plane stress system and the bending system it yields is more complicated than the Reissner-Mindlin system. In particular, the stretching boundary-value problem is a coupled system in three scalar unknowns in contrast to the two of generalized plane stress, and the bending boundary-value problem is a coupled system in four scalar unknowns in contrast to the three unknowns of the Reissner-Mindlin system. In the case of the bending problem this is referred to as the $(1,1,2)$ model in the terminology of Babuška and Li $[\mathbf{4}, \mathbf{1 2}]$.

\section{An Alternative Variational Approach}

A variant of the Hellinger-Reissner principle, which we shall call $\mathrm{HR}^{\prime}$, leads to somewhat different plate models. We shall discuss one of these models which is both more accurate and more amenable to rigorous justification than the methods based on HR discussed above. For HR' we define

$$
\underline{\underline{\Sigma}}_{\underline{g}}^{*}=\left\{\underline{\underline{\sigma}} \in \underline{\underline{H}}\left(\underline{\operatorname{div}}, P_{t}\right) \mid \underline{\underline{\sigma}} \underline{\underline{n}}=\underline{g} \text { on } \partial P_{t}^{ \pm}\right\}, \quad \underline{V}^{*}=\underline{L}^{2}(P) .
$$

The space $\underline{\underline{H}}\left(\underline{\operatorname{div}}, P_{t}\right)$ is the space of square integrable symmetric matrix-valued functions on $P_{t}$ with square integrable divergence. Then $\mathrm{HR}^{\prime}$ characterizes $\left(\underline{\underline{\sigma}}^{*}, \underline{u}^{*}\right)$ as the unique critical point (again a saddle point) of the $\mathrm{HR}^{\prime}$ functional

$$
J^{\prime}(\underline{\underline{\tau}}, \underline{v})=\frac{1}{2} \int_{P_{t}} \underline{\underline{\underline{A}}} \underline{\underline{\tau}}: \underline{\underline{\tau}} d \underline{x}+\int_{P_{t}} \underline{\operatorname{div}} \underline{\underline{\tau}} \cdot \underline{v} d \underline{x}+\int_{P_{t}} \underline{f} \cdot \underline{v} d \underline{x}
$$


TABLE 2. Summary of the principle plate models based on the second form of the Hellinger-Reissner principle.

\begin{tabular}{cccccc}
\hline model & $\operatorname{deg}_{3} \underset{\sim}{\sigma}$ & $\operatorname{deg}_{3} \underset{\sim}{\sigma}$ & $\operatorname{deg}_{3} \sigma_{33}$ & $\operatorname{deg}_{3} \underset{\sim}{\sim}$ & $\operatorname{deg}_{3} u_{3}$ \\
\hline $\operatorname{HR}_{1}^{\prime}(p)$ & $p$ & $p-1$ & $p$ & $p$ & $p-1$ \\
$\operatorname{HR}_{2}^{\prime}(p)$ & $p$ & $p+1$ & $p$ & $p$ & $p-1$ \\
$\operatorname{HR}_{3}^{\prime}(p)$ & $p$ & $p+1$ & $p$ & $p$ & $p+1$ \\
$\operatorname{HR}_{4}^{\prime}(p)$ & $p$ & $p+1$ & $p+2$ & $p$ & $p+1$ \\
\hline
\end{tabular}

on ${\underline{\underline{\sum_{g}}}}_{\underline{g}} \times \underline{V}^{*}$. Equivalently, $\left(\underline{\underline{\sigma}}^{*}, \underline{u}^{*}\right)$ is the unique element of ${\underline{\underline{\sum_{g}}}}_{\underline{g}} \times \underline{V}^{*}$ satisfying the weak equations

$$
\begin{gathered}
\int_{P_{t}} \underline{\underline{\underline{A}}} \underline{\underline{\sigma}}^{*}: \underline{\underline{\tau}} d \underline{x}+\int_{P_{t}} \underline{u} \cdot \underline{\operatorname{div}} \underline{\underline{\tau}} d \underline{x}=0 \quad \text { for all } \underline{\underline{\tau}} \in \underline{\underline{\Sigma}}_{0}^{*}, \\
\int_{P_{t}} \operatorname{div} \underline{\underline{\sigma}} \cdot \underline{v} d \underline{x}=-\int_{P_{t}} \underline{f} \cdot \underline{v} d \underline{x} \quad \text { for all } \underline{v} \in \underline{V}^{*} .
\end{gathered}
$$

Here $\underline{\underline{\Sigma}}_{0}^{*}=\left\{\underline{\underline{\sigma}} \in \underline{\underline{H}}\left(\underline{\operatorname{div}}, P_{t}\right) \mid \underline{\underline{\sigma}} \underline{\underline{n}}=0\right.$ on $\left.\partial P_{t}^{ \pm}\right\}$. Note that the displacement boundary conditions, which were essential to the first form of the Hellinger-Reissner principle, are natural in this setting, while the reverse situation holds for the traction boundary conditions.

By restricting $J^{\prime}$ to subspaces of $\underline{\underline{\Sigma}}_{g}^{*}$ and $\underline{V}^{*}$ with a specified polynomial dependence on $x_{3}$, we obtain a variety of plate models. Table 2 lists four families of models of this type. All but the second were studied (in the case of bending only) by Alessandrini in his thesis [1].

Here we shall consider only the model $\mathrm{HR}_{4}^{\prime}(1)$. For this model (and a number of others from Table 2), we have $\operatorname{div} \underline{\underline{\Sigma}}_{0}=\underline{V}$. This not only implies that there is a unique solution $(\underline{\underline{\sigma}}, \underline{u})$ but also that

$$
\operatorname{div} \underline{\underline{\sigma}}=-P_{\underline{V}} \underline{f}
$$

the orthogonal projection of $\underline{f}$ in $\underline{V}^{*}$ onto $\underline{V}$. It follows that $\underline{\underline{\sigma}}$ minimizes the complementary energy

$$
E_{c}(\underline{\underline{\tau}})=\frac{1}{2} \int_{P_{t}} \underline{\underline{\underline{A}}} \underline{\underline{\tau}}: \underline{\underline{\tau}} d \underline{x}
$$

over all $\underline{\underline{\tau}} \in \underline{\underline{\sum}}_{\underline{g}}$ satisfying the equilibrium condition $\underline{\operatorname{div}} \underline{\underline{\tau}}=-P_{\underline{V}} \underline{f}$. That is, the $\mathrm{HR}_{4}^{\prime}(1)$ model is a minimum complementary energy model.

We remark that one of Reissner's own derivation of the Reissner-Mindlin plate bending model [10] used a complementary energy approach close to $\mathrm{HR}_{4}^{\prime}(1)$. However, the trial subspace of $\stackrel{\underline{\Sigma}}{*}_{g}^{*}$ which Reissner used is not determined simply by bounding the polynomial degrees of the various components. The resulting plate bending model is very close to what we shall obtain, but the approach is not as amenable to error analysis or to generalization to higher formal order.

The $\mathrm{HR}_{4}^{\prime}(1)$ model gives

$$
\underline{u}(\underline{x})=\left(\begin{array}{c}
\underset{\sim}{\sim} \underset{\sim}{(x)} \\
\rho(\underset{\sim}{x}) x_{3}
\end{array}\right)+\left(\begin{array}{c}
-\underset{\sim}{\phi}(\underset{\sim}{x}) x_{3} \\
\omega(\underset{\sim}{x})+\underset{\omega}{\omega}(\underset{\sim}{x}) r\left(x_{3}\right)
\end{array}\right),
$$


and

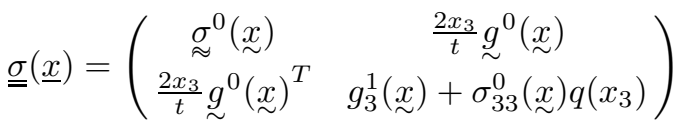

$$
\begin{aligned}
& +\left(\begin{array}{cc}
\underset{\sim}{{\underset{\sim}{\sigma}}^{1}(\underset{\sim}{x}) \frac{x_{3}}{t}} & \underset{\sim}{g^{1}}(\underset{\sim}{x})+{\underset{\sim}{\sigma}}^{0}(\underset{\sim}{x}) q\left(x_{3}\right) \\
\underset{\sim}{g^{1}}(\underbrace{x}_{\sim})^{T}+\underset{\sim}{\sigma^{0}}(\underset{\sim}{x})^{T} q\left(x_{3}\right) & g_{3}^{0}(\underset{\sim}{x}) \frac{x_{3}}{t}+\sigma_{33}^{1}\left(x_{3}\right) s\left(x_{3}\right)
\end{array}\right),
\end{aligned}
$$

where the coefficient functions $\underset{\sim}{\eta}, \rho, \underset{\sim}{\phi}, \omega, \omega_{2}, \underset{\approx}{\sigma^{0}}, \sigma_{33}^{0}, \underset{\approx}{\sigma^{1}},{\underset{\sim}{\sigma}}^{0}$, and $\sigma_{33}^{1}$ are functions of $\underset{\sim}{x}$ which we shall describe, and the polynomials $q, r$, and $s$ are given by $q(z)=3 / 2-6 z^{2} / t^{2}, r(z)=6 z^{2} / t^{2}-3 / 10$, and $s(z)=(5 / 2) z / t-10 z^{3} / t^{3}$. In the appendix we verify that the coefficient functions are determined by the following 2-D boundary-value problems.

The stretching solution. The stretching portion of the solution is determined by the solution to the boundary-value problem

$$
\begin{aligned}
& -t \operatorname{div}_{\sim} \underset{\approx}{A^{-1}} \underset{\sim}{\varepsilon}(\eta)=\underset{\sim}{l}+t \frac{\nu}{1-\nu} \underset{\sim}{\nabla} l_{2} \text { in } \Omega, \\
& \underset{\sim}{\eta}=0 \text { on } \partial \Omega,
\end{aligned}
$$

where

$$
\underset{\sim 1}{l}=2 \underset{\sim}{g^{0}}+{\underset{\sim}{f}}^{0}, \quad l_{2}=g_{3}^{1}+\frac{t}{6} \operatorname{div} \underset{\sim}{g^{0}}+f_{3}^{1} .
$$

Note that only the loading term $\underset{\sim}{\stackrel{l}{l}}$ appeared in the $\mathrm{HR}_{1}(1)$ model described earlier. This model takes into account the effect of an odd transverse load on stretching, which is ignored in $\mathrm{HR}_{1}(1)$.

With $\underset{\sim}{\eta}$ uniquely determined by (14)-(15), the remaining solution quantities are given by

$$
\begin{gathered}
{\underset{\sim}{\sigma}}^{0}=\underset{\approx}{\left.A^{-1} \underset{\sim}{\underset{\sim}{\varepsilon}} \underset{\sim}{\eta}\right)+\frac{\nu}{1-\nu} l_{2} \underset{\sim}{\delta},} \\
\sigma_{33}^{0}=\frac{t}{6} \operatorname{div} \underset{\sim}{g^{0}}+f_{3}^{1}, \\
\rho=\frac{1}{E}\left[-\nu \operatorname{tr}\left({\underset{\sim}{\sigma}}^{0}\right)+\frac{6}{5} \sigma_{33}^{0}+g_{3}^{1}\right] .
\end{gathered}
$$

The bending solution. The bending portion of the solution is determined by the solution to the boundary-value problem

$$
\begin{gathered}
\left.-\frac{t^{3}}{12} \operatorname{div}_{\sim} \underset{\approx}{A_{\approx}^{-1}} \underset{\sim}{\underset{\sim}{\varepsilon}} \underset{\sim}{\phi}\right)+t \frac{5}{6} \frac{E}{2(1+\nu)}(\underset{\sim}{\phi}-\underset{\sim}{\nabla} \omega)=t \underset{\sim}{k}-\frac{t^{2}}{12} \underset{\sim}{\nabla} k_{2} \text { in } \Omega, \\
t \frac{5}{6} \frac{E}{2(1+\nu)} \operatorname{div}(\underset{\sim}{\phi}-\underset{\sim}{\nabla} \omega)=k_{3} \text { in } \Omega, \\
\underset{\sim}{\phi}=0, \quad \omega=0 \text { on } \partial \Omega,
\end{gathered}
$$


where

$$
\begin{aligned}
& \underset{\sim}{k_{1}}=-\frac{5}{6} g_{\sim}^{1}-{\underset{\sim}{f}}^{1}, \quad k_{2}=\frac{\nu}{1-\nu}\left[\frac{t}{5} \operatorname{div} \underset{\sim}{g^{1}}+\frac{12}{5} g_{3}^{0}+f_{3}^{2}\right], \\
& k_{3}=\frac{t}{6} \operatorname{div} \underset{\sim}{g}{ }^{1}+2 g_{3}^{0}+f_{3}^{0} \text {. }
\end{aligned}
$$

Here we have introduced the quantity $f_{3}^{2}(\underset{\sim}{x})=\int_{-t / 2}^{t / 2} f_{3}(\underline{x}) r\left(x_{3}\right) d x_{3}$.

The equations (18)-(19) are a somewhat different version of the ReissnerMindlin equations than (6)-(7), which arose from the $\mathrm{HR}_{1}(1)$ model. Not only are the formulas for the applied load and couple more involved, but a shear correction factor of $5 / 6$ has been introduced.

With $\underset{\sim}{\phi}$ and $\omega$ determined by $(18)-(20)$ we find

$$
\begin{aligned}
& \sigma_{\approx}^{1}=-t \underset{\approx}{A_{\approx}^{-1}} \underset{\sim}{\varepsilon}(\phi)+k_{2} \delta, \\
& \sigma_{\sim}^{0}=\frac{5}{6}\left[\frac{E}{2(1+\nu)}(-\underset{\sim}{\phi}+\underset{\sim}{\nabla} \omega)-{\underset{\sim}{g}}^{1}\right], \\
& \sigma_{33}^{1}=\frac{t}{5} \operatorname{div} \underset{\sim}{g}{ }^{1}+\frac{2}{5} g_{3}^{0}+f_{3}^{2}, \\
& \omega_{2}=\frac{t}{E}\left[\frac{1}{6} g_{3}^{0}+\frac{5}{42} \sigma_{33}^{1}-\frac{\nu}{12} \operatorname{tr}\left(\underset{\approx}{\sigma^{1}}\right)\right] \text {. }
\end{aligned}
$$

\section{Bounding the Modelling Error}

An important advantage of the $\mathrm{HR}_{4}^{\prime}(1)$ method is that it is amenable to rigorous error analysis. To state our main result, we make the following assumptions on the data $\underline{f}$ and $\underline{g}$. We assume that $\underline{f} \in \underline{V}$, i.e., that $\underset{\sim}{f}$ varies linearly with $x_{3}$ and $f_{3}$ quadratically, and that $f_{3}^{i} \in H^{1}(\Omega), i=1,2,3$. We assume that $g \in \underline{L}^{2}\left(\partial P_{t}^{ \pm}\right)$and also that $\operatorname{div} \underset{\sim}{g}, g_{3} \in H^{1}\left(\partial P_{t}^{ \pm}\right)$. For such loading functions we define the norms

$$
\begin{gathered}
N\left({\underset{\sim}{f}}^{0}\right)=\left\|{\underset{\sim}{f}}^{0}\right\|_{0}, \quad N\left({\underset{\sim}{f}}^{1}\right)=\left\|{\underset{\sim}{f}}^{1}\right\|_{0}, \\
N\left(f_{3}^{0}, f_{3}^{2}\right)=\left\|f_{3}^{0}\right\|\left\|_{-1}+t^{3 / 2}\right\| f_{3}^{2}\left\|_{0}+t^{2}\right\| f_{3}^{2}\left\|_{1}, \quad N\left(f_{3}^{1}\right)=\right\| f_{3}^{1}\left\|_{0}+t^{1 / 2}\right\| f_{3}^{1} \|_{1}, \\
N\left(\underset{\sim}{g^{0}}\right)=\left\|\underset{\sim}{g^{0}}\right\|_{0}+t^{3 / 2}\left\|\operatorname{div} \underset{\sim}{g^{0}}\right\|_{0}+t^{2}\left\|\operatorname{div} \underset{\sim}{g^{0}}\right\|_{1}, \\
N\left(\underset{\sim}{g^{1}}\right)=\left\|\underset{\sim}{g^{1}}\right\|_{0}+t^{3 / 2}\left\|\operatorname{div} \underset{\sim}{g^{1}}\right\|_{0}+t^{2}\|\operatorname{div} \underset{\sim}{g}\|_{1}, \\
N\left(g_{3}^{0}\right)=\left\|g_{3}^{0}\right\|_{-1}+t^{3 / 2}\left\|g_{3}^{0}\right\|_{0}+t^{2}\left\|g_{3}^{0}\right\|_{1}, \quad N\left(g_{3}^{1}\right)=\left\|g_{3}^{1}\right\|_{1} .
\end{gathered}
$$

The precise form of these $t$-dependent norms is unessential. The key point is that if the functions $f^{i}, f_{3}^{i}, g^{i}, g_{3}^{i}$ on $\Omega$ are nonzero smooth functions independent of $t$, then the norms given tend to a finite nonzero limit as $t$ tends to zero.

Theorem 1. Let $t \in(0,1]$ and suppose that $f: P_{t} \rightarrow \mathbb{R}^{3}$ and $g: \partial P_{t}^{ \pm} \rightarrow \mathbb{R}^{3}$ satisfy the conditions just stated. Define $\left(\underline{\underline{\sigma}}^{*}, \underline{u}^{*}\right)$ as the solution to the threedimensional elasticity problem (1) and define $(\underline{\underline{\sigma}}, \underline{u})$ as the solution to the $\mathrm{HR}_{4}^{\prime}(1)$ 
model (12)-(22). Then we have

$$
\begin{aligned}
\left\|\underline{\underline{\varepsilon}}\left(\underline{u}^{*}-\underline{u}\right)\right\|_{0, P_{t}}+\left\|\underline{\underline{\sigma}}^{*}-\underline{\underline{\sigma}}\right\|_{0, P_{t}} \leq & C\left[N\left({\underset{\sim}{g}}^{0}\right)+N\left({\underset{\sim}{f}}^{0}\right)+t N\left(g_{3}^{1}\right)+t^{1 / 2} N\left(f_{3}^{1}\right)\right. \\
& \left.+t^{-1} N\left(g_{3}^{0}\right)+t^{-1} N\left(f_{3}^{0}, f_{3}^{2}\right)+N\left(\underline{\sim}^{1}\right)+N\left({\underset{\sim}{f}}^{1}\right)\right],
\end{aligned}
$$

where $C$ is a constant independent of $t, \underline{g}$, and $\underline{f}$.

Before proceeding to the proof of the theorem, we discuss its significance. First we consider a simple case of pure stretching, namely that where the surface load is purely in-plane and even in $x_{3}$, and the volume load vanishes. That is, suppose that for each $t$,

$$
\underline{g}(\underset{\sim}{x}, t / 2)=\underline{g}(\underset{\sim}{x},-t / 2)=\left(\begin{array}{c}
{\underset{\sim}{0}}^{0}(\underset{\sim}{x}) \\
0
\end{array}\right),
$$

with $\underset{\sim}{g^{0}}$ a smooth function on $\Omega$ independent of $t$, and that $\underline{f}$ is identically zero. Then $\stackrel{\sim}{\text { Theorem }} 1$ gives the estimate

$$
\left\|\underline{\underline{\varepsilon}}\left(\underline{u}^{*}-\underline{u}\right)\right\|_{0, P_{t}}+\left\|\underline{\underline{\sigma}}^{*}-\underline{\underline{\sigma}}\right\|_{0, P_{t}} \leq \mathrm{const} .
$$

with the constant depending on $g$ but not on $t$. However, from (14)-(17), we easily see that $\|\underset{\sim}{\varepsilon}(\underset{\sim}{\eta})\|_{0}$ and $\left\|{\underset{\sim}{\sigma}}^{0}\right\|_{0}$ behave as $O\left(t^{-1}\right)$ as $t$ tends to zero, and consequently $\|\underline{\underline{\varepsilon}}(\underline{u})\|_{0, P_{t}}$ and $\|\underline{\underline{\sigma}}\|_{0, P_{t}}$ behave as $O\left(t^{-1 / 2}\right)$. Thus we find that the relative error

$$
\frac{\left\|\underline{\underline{\varepsilon}}\left(\underline{u}^{*}-\underline{u}\right)\right\|_{0, P_{t}}}{\|\underline{\underline{\varepsilon}}(\underline{u})\|_{0, P_{t}}}+\frac{\left\|\underline{\underline{\sigma^{*}}}-\underline{\underline{\sigma}}\right\|_{0, P_{t}}}{\|\underline{\underline{\sigma}}\|_{0, P_{t}}} \leq C t^{1 / 2} .
$$

In other words, the plate model converges with order $t^{1 / 2}$. Note that we could have as well assumed that the surface load was proportional to a function of $t$, rather than independent of $t$, and still have obtained $O\left(t^{1 / 2}\right)$ convergence. Indeed, for the relative error, any such factor cancels out.

In the case of a purely in-plane and even volume load and vanishing surface load, we obtain the same result in essentially the same way.

Next consider the simplest case of pure bending, namely a transverse, even surface load and no volume load:

$$
\underline{g}(\underset{\sim}{x}, t / 2)=\underline{g}(\underset{\sim}{x},-t / 2)=\left(\begin{array}{c}
0 \\
g_{3}^{0}(\underset{\sim}{x})
\end{array}\right) .
$$

In this case the theorem gives

$$
\left\|\underline{\underline{\varepsilon}}\left(\underline{u}^{*}-\underline{u}\right)\right\|_{0, P_{t}}+\left\|\underline{\underline{\sigma}}^{*}-\underline{\underline{\sigma}}\right\|_{0, P_{t}} \leq C t^{-1} .
$$

But from (18)-(22) we see that $\|\underset{\sim}{\phi}\|_{1}$ behaves as $O\left(t^{-3}\right)$ and $\left\|{\underset{\sim}{\sigma^{1}}}^{1}\right\|_{0}$ as $O\left(t^{-2}\right)$. Thus $\|\underline{\underline{\varepsilon}}(\underline{u})\|_{0, P_{t}}$ and $\|\underline{\underline{\sigma}}\|_{0, P_{t}}$ behave as $O\left(t^{-3 / 2}\right)$, and again we have a convergence rate of $O\left(t^{1 / 2}\right)$ in relative energy norm. The same results holds for bending induced by a transverse, even volume load.

A less common situation is when bending is induced by an in-plane surface load which acts in opposite directions on the top and bottom surfaces:

$$
\underline{g}(\underset{\sim}{x}, t / 2)=-\underline{g}(\underset{\sim}{x},-t / 2)=\left(\begin{array}{c}
\left.{\underset{\sim}{1}}^{1} \underset{\sim}{x}\right) \\
0
\end{array}\right) .
$$


Once again we obtain a relative error convergence rate of $O\left(t^{1 / 2}\right)$. The same result holds for bending induced by an odd in-plane volume loading, and for stretching induced by an odd transverse surface loading.

We are aware of one case where $O\left(t^{1 / 2}\right)$ convergence does not hold. Suppose that the surface load vanishes and the volume load is transverse and odd in $x_{3}$ :

$$
\underline{f}(\underline{x})=\left(\begin{array}{c}
0 \\
f(\underset{\sim}{x}) x_{3}
\end{array}\right),
$$

where $f$ is non-constant. Then $f_{3}^{1}=t^{2} f / 12$, while the other load quantities vanish. Therefore Theorem 1 gives

$$
\left\|\underline{\underline{\varepsilon}}\left(\underline{u}^{*}-\underline{u}\right)\right\|_{0, P_{t}}+\left\|\underline{\underline{\sigma}}^{*}-\underline{\underline{\sigma}}\right\|_{0, P_{t}} \leq C t^{5 / 2},
$$

but $\|\underline{\underline{\varepsilon}}(\underline{u})\|_{0, P_{t}}$ and $\|\underline{\underline{\sigma}}\|_{0, P_{t}}$ are $O\left(t^{5 / 2}\right)$ in this case as well. Thus, in this loading case, we don't see any convergence. (We remark that this exceptional situation doesn't pertain to a transverse volume load due to gravity, since then its odd part vanishes.)

\section{Proof of the Modelling Error Bounds}

The proof of Theorem 1 will be based on the two energies principle or PragerSynge theorem $[\mathbf{9}]$. To state this principle, we define the energy norm of $\underline{v} \in \underline{H}^{1}\left(P_{t}\right)$ by

$$
\|\underline{v}\|_{\mathrm{e}}^{2}=\int_{P_{t}} \underline{\underline{\underline{A}}}^{-1} \underline{\underline{\varepsilon}}(\underline{v}): \underline{\underline{\varepsilon}}(\underline{v}) d \underline{x},
$$

and the complementary energy norm of $\underline{\underline{\tau}} \in \underline{\underline{L}}^{2}\left(P_{t}\right)$ by

$$
\|\underline{\underline{\tau}}\|_{c}^{2}=\int_{P_{t}} \underline{\underline{\underline{A}}} \underline{\underline{\underline{\tau}}}: \underline{\underline{\tau}} d \underline{x} \text {. }
$$

Clearly, $\|\underline{v}\|_{\mathrm{e}}$ is equivalent to $\|\underline{\underline{\varepsilon}}(\underline{v})\|_{0, P_{t}}$ and $\|\underline{\underline{\tau}}\|_{\mathrm{c}}$ is equivalent to $\|\underline{\underline{\tau}}\|_{0, P_{t}}$ with equivalence constants independent of $t$. The form of the two energies principle we use states that if a stress field and displacement field satisfy the equilibrium equation and boundary conditions of the three-dimensional elasticity problem, then the energy norm of their difference from the true solution of the $3 \mathrm{D}$ problem can be written in terms of a norm of the residual of the constitutive equation.

Theorem 2. Suppose that $\underline{\underline{\underline{\sigma}}} \in \underline{\underline{H}}\left(\underline{\operatorname{div}}, P_{t}\right)$ satisfies

$$
-\operatorname{div} \underline{\underline{\bar{\sigma}}}=\underline{f} \text { in } P_{t}, \quad \underline{\underline{\sigma}} \underline{n}=\underline{g} \text { on } \partial P_{t}^{ \pm},
$$

and that $\underline{\bar{u}} \in \underline{H}^{1}\left(P_{t}\right)$ satisfies

$$
\underline{\bar{u}}=0 \text { on } \partial P_{t}^{\mathrm{L}} \text {. }
$$

Then

$$
\left\|\underline{u}^{*}-\underline{\bar{u}}\right\|_{\mathrm{e}}^{2}+\left\|\underline{\underline{\sigma}}^{*}-\underline{\underline{\sigma}}\right\|_{\mathrm{c}}^{2}=\left\|\underline{\underline{\bar{\sigma}}}-\underline{\underline{\underline{\underline{A}}}}^{-1} \underline{\underline{\varepsilon}}(\underline{\bar{u}})\right\|_{\mathrm{c}}^{2} .
$$

Since $\underline{\underline{\sigma}}$ belongs to $\underline{\underline{\sum_{g}}}$ and satisfies (11), and because we assumed that $\underline{f} \in \underline{V}$, we may choose $\underline{\underline{\bar{\sigma}}}=\underline{\underline{\sigma}}$ and $(23)$ is satisfied. We may not, however, take $\underline{\bar{u}}=\underline{u}$, because, 
in general, the third component of $\underline{u}$ does not vanish on the lateral boundary and so (24) would not be satisfied.

Therefore we define

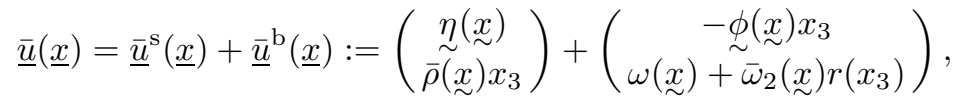

where $\bar{\rho}$ and $\bar{\omega}_{2}$ are functions in $\stackrel{\circ}{H}^{1}(\Omega)$ which are yet to be defined. We will use the Prager-Synge theorem to bound $\left\|\underline{\underline{\varepsilon}}\left(\underline{u}^{*}-\underline{\bar{u}}\right)\right\|_{0, P_{T}}$ and $\left\|\underline{\underline{\sigma}}^{*}-\underline{\underline{\sigma}}\right\|_{0, P_{T}}$, and then bound $\left\|\underline{\underline{\varepsilon}}\left(\underline{u}^{*}-\underline{u}\right)\right\|_{0, P_{T}}$ via the triangle inequality.

A priori estimates. The first step is to derive some a priori estimates for the plate model.

Stretching. The following lemma summarizes the regularity of quantities associated to the stretching problem.

Lemma 3. Let $s \geq 0$ be an integer. There exist a constant $C$ depending only on $s, \Omega, E$, and $\nu$ such that

$$
\left\|\sigma_{33}^{0}\right\|_{s} \leq C\left(t\|\operatorname{div} \underset{\sim}{g}\|_{s}+\left\|f_{3}^{1}\right\|_{s}\right),
$$

and

$$
\begin{array}{r}
\|\underset{\sim}{\eta}\|_{s+1}+\left\|\underset{\sim}{\sigma^{0}}\right\|_{s}+\|\rho\|_{s} \leq C\left(t^{-1}\|\underset{\sim}{g}\|_{s-1}+t^{-1}\|\underset{\sim}{f}\|_{s-1}+\left\|g_{3}^{1}\right\|_{s}\right. \\
\left.[t]+\left\|f_{3}^{1}\right\|_{s}+t\left\|\operatorname{div} \underset{\sim}{g^{0}}\right\|_{s}\right) .
\end{array}
$$

Proof. The estimate for $\sigma_{33}^{0}$ is immediate from its definition (17) (with $C=$ $1)$. The estimate for $\underset{\sim}{\eta}$ follows directly from standard regularity estimates for the plane elasticity problem $(14)-(15)$, and then the estimates for $\underset{\approx}{\sigma}$ and $\rho$ follow from their definitions.

Bending. For the bending problem, the situation is more complicated, because the boundary-value problem (18)-(20) involves a boundary layer. The following result is proved in $[\mathbf{2}]$.

THEOREM 4. Let $\Omega$ be a convex polygon or a smoothly bounded domain in the plane. For any $t \in(0,1], \underset{\sim}{F} \in \underset{\sim}{H^{-1}}(\Omega)$, and $G \in H^{-1}(\Omega)$, there exists a unique $(\underset{\sim}{\phi}, \omega) \in \underset{\sim}{H^{1}}(\Omega) \times H^{1}(\Omega)$ such that

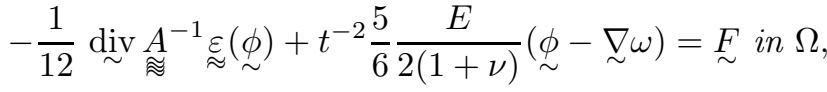

$$
\begin{aligned}
& t^{-2} \frac{5}{6} \frac{E}{2(1+\nu)} \operatorname{div}(\underset{\sim}{\phi}-\underset{\sim}{\nabla} \omega)=G \text { in } \Omega, \\
& \phi=0, \omega=0 \text { on } \partial \Omega .
\end{aligned}
$$

Moreover, if $\underset{\sim}{F} \in L^{2}(\Omega)$, then $\underset{\sim}{\phi} \in H^{2}(\Omega)$ and there exists a constant $C$ independent of $t, \underset{\sim}{F}$, and $G$, such that

$$
\|\underset{\sim}{\phi}\|_{2}+\|\omega\|_{1}+t^{-2}\|\underset{\sim}{\nabla} \omega-\underset{\sim}{\phi}\|_{0} \leq C\left(\|\underset{\sim}{F}\|_{0}+\|G\|_{-1}\right) .
$$


We note that the estimate for $\underset{\sim}{\nabla} \omega-\underset{\sim}{\phi}$ follows directly from that for $\underset{\sim}{\phi}$ and the first differential equation. We also remark that this result does not hold for some other common boundary conditions for the Reissner-Mindlin plate, due to the presence of a stronger boundary layer. For such problems a more refined analysis, based on the asymptotic analysis of $[\mathbf{3}]$, is needed.

LEMMA 5.

(1) For any $s$

$$
\left\|\sigma_{33}^{1}\right\|_{s} \leq t\left\|\operatorname{div} \underset{\sim}{g^{1}}\right\|_{s}+\left\|g_{3}^{0}\right\|_{s}+\left\|f_{3}^{2}\right\|_{s} .
$$

(2) There exist a constant $C$ depending only on $\Omega, E$, and $\nu$ such that

$$
\begin{aligned}
& \|\underset{\sim}{\phi}\|_{2}+\|\omega\|_{1}+t^{-2}\|\underset{\sim}{\nabla} \omega-\underset{\sim}{\phi}\|_{0}+t^{-1}\left\|\underset{\sim}{\sigma^{1}}\right\|_{1}+t^{-2}\left\|\omega_{2}\right\|_{1} \\
& \quad \leq C t^{-3}\left(\left\|g_{3}^{0}\right\|_{-1}+t^{2}\left\|g_{3}^{0}\right\|_{1}+\left\|f_{3}^{0}\right\|_{-1}+t^{2}\left\|f_{3}^{2}\right\|_{1}+t\left\|\underset{\sim}{g^{1}}\right\|_{0}+t^{3}\left\|\operatorname{div} \underset{\sim}{g^{1}}\right\|_{1}+t\left\|f_{\sim}^{1}\right\|_{0}\right) .
\end{aligned}
$$

Proof. The estimate for $\sigma_{33}^{1}$ is immediate from its definition (22). The estimates for $\phi, \omega$, and $\underset{\sim}{\nabla} \omega-\phi$ follow directly from Theorem 4 by taking $\underset{\sim}{F}=$ $t^{-2} \underset{\sim}{k_{1}}-t^{-1} \underset{\sim}{\sim} k_{2} / 12$ and $G=\tilde{t}^{-3} k_{3}$, and the estimates for $\underset{\sim}{\sigma^{1}}$ and $\omega_{2}$ then follow directly from (22).

Estimation of the residual in the constitutive equation. We now proceed to the estimation of $\|\underline{\underline{\underline{\underline{A}}}} \underline{\underline{\underline{\sigma}}}-\underline{\underline{\varepsilon}}(\underline{\bar{u}})\|_{0, P_{t}}$ (which is equivalent to $\left\|\underline{\underline{\sigma}}-\underline{\underline{\underline{\underline{A}}}}^{-1} \underline{\underline{\varepsilon}}(\underline{\bar{u}})\right\|_{\mathrm{c}}$ ).

Stretching. The form of $\underline{\underline{\sigma}}^{\mathrm{s}}$ and $\underline{\underline{u}}^{\mathrm{s}}$ give

$$
\underline{\underline{\underline{\underline{A}}}} \underline{\underline{\underline{\sigma}}}^{\mathrm{s}}=\left(\begin{array}{cc}
\underset{\approx}{\approx \sigma^{0}}-\frac{\nu}{E}\left(g_{3}^{1}+\sigma_{33}^{0} q\right) \underset{\approx}{\delta} & \frac{1+\nu}{E} \frac{2 x_{3}}{t} g^{0} \\
\frac{1+\nu}{E} \frac{2 x_{3}}{t} \underline{\sim}^{0 T} & \frac{1}{E}\left(g_{3}^{1}+\sigma_{33}^{0} q\right)-\frac{\nu}{E} \operatorname{tr}\left({\underset{\approx}{\sigma}}^{0}\right)
\end{array}\right),
$$

and

$$
\underline{\underline{\varepsilon}}\left(\underline{\bar{u}}^{\mathrm{S}}\right)=\left(\begin{array}{cc}
\underset{\sim}{\varepsilon}(\underset{\eta}{\eta}) & (\underset{\sim}{\nabla} \bar{\rho}) \frac{x_{3}}{2} \\
(\underset{\sim}{\nabla} \bar{\rho})^{T} \frac{x_{3}}{2} & \bar{\rho}
\end{array}\right)
$$

which, combined with the formulas for $\sigma_{\sim}^{0}, \sigma_{33}^{0}$, and $\rho$ above, give

$$
\underline{\underline{\underline{\underline{A}}}} \underline{\underline{\sigma}}^{\mathrm{s}}-\underline{\underline{\varepsilon}}\left(\underline{\bar{u}}^{\mathrm{s}}\right)=\left(\begin{array}{cc}
\frac{\nu}{E} \sigma_{33}^{0}(1-q) \underset{\approx}{\delta} & \frac{1+\nu}{E} \frac{2 x_{3}}{t} g^{0}-(\underset{\sim}{\nabla} \bar{\rho}) \frac{x_{3}}{2} \\
\frac{1+\nu}{E} \frac{2 x_{3}}{t} \underline{\sim}^{0 T}-(\underset{\sim}{\nabla} \bar{\rho})^{T} \frac{x_{3}}{2} & \rho-\bar{\rho}+\frac{1}{E} \sigma_{33}^{0}(q-6 / 5)
\end{array}\right) .
$$

It follows that

$$
\left\|\underline{\underline{\underline{\underline{A}}}} \underline{\underline{\underline{\sigma}}}^{\mathrm{s}}-\underline{\underline{\varepsilon}}\left(\underline{\bar{x}}^{\mathrm{s}}\right)\right\|_{0, P_{t}}^{2} \leq C t\left(\left\|{\underset{\sim}{g}}^{0}\right\|_{0}^{2}+\left\|\sigma_{33}^{0}\right\|_{0}^{2}+t^{2}\|\underset{\sim}{\nabla} \bar{\rho}\|_{0}^{2}+\|\bar{\rho}-\rho\|_{0}^{2}\right) .
$$

In view of this estimate we choose $\bar{\rho}$ as the $\stackrel{\circ}{H}^{1}(\Omega)$ function which minimizes

$$
t^{2}\|\underset{\sim}{\nabla}\|_{0}^{2}+\|\bar{\rho}-\rho\|_{0}^{2},
$$

or, equivalently, as the solution of the boundary-value problem

$$
-t^{2} \Delta \bar{\rho}+\bar{\rho}=\rho \text { in } \Omega, \quad \bar{\rho}=0 \text { on } \partial \Omega .
$$

We then have the following estimate. 
Lemma 5. Let $\rho \in H^{1}(\Omega)$ and let $\bar{\rho}$ be the solution of $(27)$. Then

$$
t^{2}\|\underset{\sim}{\nabla}\|_{0}^{2}+\|\bar{\rho}-\rho\|_{0}^{2} \leq C\left(t\|\rho\|_{0, \partial \Omega}^{2}+t^{2}\|\rho\|_{1}^{2}\right) .
$$

Proof. Multiplying the differential equation by $-\Delta \bar{\rho}$ and integrating by parts gives

$$
t^{2}\|\Delta \bar{\rho}\|_{0}^{2}+\|\underset{\sim}{\nabla} \bar{\rho}\|_{0}^{2}=\int_{\Omega} \underset{\sim}{\nabla} \rho \cdot \underset{\sim}{\nabla} \bar{\rho} d \underset{\sim}{x}-\int_{\partial \Omega} \rho \frac{\partial \bar{\rho}}{\partial n} d s .
$$

Now

$$
\|\partial \bar{\rho} / \partial n\|_{0, \partial \Omega}^{2} \leq C\|\bar{\rho}\|_{1}\|\bar{\rho}\|_{2} \leq C\left(t^{-1}\|\bar{\rho}\|_{1}^{2}+t\|\bar{\rho}\|_{2}^{2}\right),
$$

so, for any $\epsilon>0$ we have

$$
\left|\int_{\partial \Omega} \rho \frac{\partial \bar{\rho}}{\partial n} d s\right| \leq C_{\epsilon} t^{-1}\|\rho\|_{0, \partial \Omega}^{2}+\epsilon\left(\|\bar{\rho}\|_{1}^{2}+t^{2}\|\bar{\rho}\|_{2}^{2}\right) .
$$

Clearly also

$$
|(\underset{\sim}{\nabla} \rho, \underset{\sim}{\nabla} \bar{\rho})| \leq C_{\epsilon}\|\rho\|_{1}^{2}+\epsilon\|\bar{\rho}\|_{1}^{2} .
$$

It follows easily from these estimates that

$$
t^{2}\|\Delta \bar{\rho}\|_{0}^{2}+\|\underset{\sim}{\nabla}\|_{0}^{2} \leq C\left(t^{-1}\|\rho\|_{0, \partial \Omega}^{2}+\|\rho\|_{1}^{2}\right) .
$$

Multiplying by $t^{2}$ and making the substitution $t^{2} \Delta \bar{\rho}=\bar{\rho}-\rho$ gives the lemma.

Applying the lemma to (26) and bounding $\|\rho\|_{0, \partial \Omega}$ by $\|\rho\|_{1}$, we get

$$
\begin{aligned}
\left\|\underline{\underline{\underline{\underline{A}}}} \underline{\underline{\underline{\sigma}}}^{\mathrm{s}}-\underline{\underline{\varepsilon}}\left(\underline{\underline{u}}^{\mathrm{s}}\right)\right\|_{0, P_{t}} \leq C t^{1 / 2}\left(\left\|{\underset{\sim}{g}}^{0}\right\|_{0}+\left\|\sigma_{33}^{0}\right\|_{0}+t^{1 / 2}\|\rho\|_{1}\right) \\
\leq C\left(\left\|{\underset{\sim}{g}}^{g}\right\|_{0}+t^{3 / 2}\left\|\operatorname{div} \underset{\sim}{g^{0}}\right\|_{0}+t^{2}\left\|\operatorname{div} \underset{\sim}{g^{0}}\right\|_{1}\right. \\
\left.\quad+\left\|\underset{\sim}{f^{0}}\right\|_{0}+t\left\|g_{3}^{1}\right\|_{1}+t^{1 / 2}\left\|f_{3}^{1}\right\|_{0}+t\left\|f_{3}^{1}\right\|_{1}\right),
\end{aligned}
$$

where we have used Lemma 3 in the last step.

REMARK. If we use the formula (17) for $\rho$ we can compute

$$
\begin{aligned}
\left\|\underline{\underline{\underline{\underline{A}}}}{\underline{\underline{\sigma^{\mathrm{s}}}}}^{\mathrm{s}}-\underline{\underline{\varepsilon}}\left(\underline{u}^{\mathrm{s}}\right)\right\|_{0, P_{t}} \leq C t^{1 / 2}\left(\left\|{\underset{\sim}{g}}^{0}\right\|_{0}+t\left\|\operatorname{div} \underset{\sim}{g^{0}}\right\|_{0}+t^{2}\left\|\operatorname{div} \underset{\sim}{g}{ }^{0}\right\|_{1}+\|\underbrace{f^{0}}_{\sim}\|_{0}\right. \\
\left.+t\left\|g_{3}^{1}\right\|_{1}+\left\|f_{3}^{1}\right\|_{0}+t\left\|f_{3}^{1}\right\|_{1}\right) .
\end{aligned}
$$

Thus this quantity is (for most loading conditions) one half order of $t$ smaller than $\left\|\underline{\underline{\underline{\underline{A}}}} \underline{\underline{\mathrm{S}}}^{\mathrm{s}}-\underline{\underline{\varepsilon}}\left(\underline{\underline{u}}^{\mathrm{s}}\right)\right\|_{0, P_{t}}$. This suggests, correctly, that if we consider a different boundaryvalue problem, such as that for a periodic plate, in which the plate model does not need to be corrected to satisfy the conditions on the lateral boundary, then the model will approximate the three dimensional solution to higher order.

For later use we note one other consequence of Lemma 6. From (25) and the analogous formula for $\underline{\underline{\varepsilon}}\left(\underline{u}^{\mathrm{s}}\right)$ we have

$$
\begin{aligned}
& \left\|\underline{\underline{\varepsilon}}\left(\underline{\bar{u}}^{\mathrm{S}}\right)-\underline{\underline{\varepsilon}}\left(\underline{u}^{\mathrm{s}}\right)\right\|_{0, P_{t}} \leq C t^{1 / 2}\left[t\|\underset{\sim}{\nabla}(\bar{\rho}-\rho)\|_{0}+\|\bar{\rho}-\rho\|_{0}\right] \\
& \leq C t^{1 / 2}\left(t\|\underset{\sim}{\nabla} \bar{\rho}\|_{0}+t\|\underset{\sim}{\nabla} \rho\|_{0}+\|\bar{\rho}-\rho\|_{0}\right) \\
& \leq C t\|\rho\|_{1} \text {. }
\end{aligned}
$$


DERIVATION AND JUSTIFICATION OF PLATE MODELS BY VARIATIONAL METHODS 15

In view of Lemma 3 we conclude

$$
\left\|\underline{\underline{\varepsilon}}\left(\underline{\bar{u}}^{\mathrm{s}}\right)-\underline{\underline{\varepsilon}}\left(\underline{u}^{\mathrm{s}}\right)\right\|_{0, P_{t}} \leq C\left(\left\|{\underset{\sim}{g}}^{0}\right\|_{0}+t^{2}\left\|\operatorname{div}{\underset{\sim}{g}}^{0}\right\|_{1}+\left\|{\underset{\sim}{f}}^{0}\right\|_{0}+t\left\|g_{3}^{1}\right\|_{1}+t\left\|f_{3}^{1}\right\|_{1}\right)
$$

Bending. Since

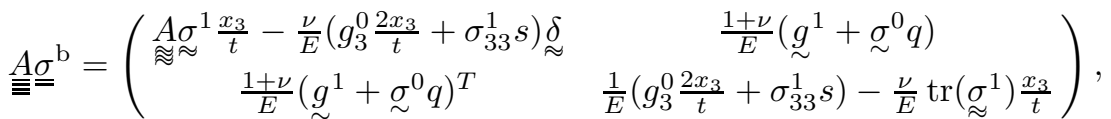

and

$$
\underline{\underline{\varepsilon}}\left(\underline{\bar{u}}^{\mathrm{b}}\right)=\left(\begin{array}{cc}
-\underset{\sim}{\varepsilon}(\phi) x_{3} & \frac{1}{2}\left[\underset{\sim}{\nabla} \omega+\left(\underset{\sim}{\nabla} \bar{\omega}_{2}\right) r-\underset{\sim}{\phi}\right] \\
\frac{1}{2}\left[\underset{\sim}{\nabla} \omega+\left(\underset{\sim}{\nabla} \bar{\omega}_{2}\right) r-\phi\right]^{T} & \bar{\omega}_{2} \frac{12 x_{3}}{t^{2}}
\end{array}\right),
$$

we obtain, in view of the formulas for $\underset{\approx}{\sigma^{1}},{\underset{\sim}{\sigma}}^{0}$, and $\omega_{2}$,

$$
\underline{\underline{\underline{\underline{A}}}} \underline{\underline{\sigma}}^{\mathrm{b}}-\underline{\underline{\varepsilon}}\left(\underline{\bar{u}}^{\mathrm{b}}\right)=\left(\begin{array}{cc}
\frac{\nu}{E} \sigma_{33}^{1}\left(\frac{x_{3}}{t}-s\right) \underset{\approx}{\delta} & \underset{\sim}{\zeta} \\
\stackrel{\sim}{\zeta}^{T} & \zeta
\end{array}\right)
$$

with

$$
\begin{aligned}
\underset{\sim}{\zeta} & =\left(\frac{1}{2}-\frac{5}{12} q\right)\left[\frac{2(1+\nu)}{E} g_{\sim}^{1}-\underset{\sim}{\nabla} \omega+\underset{\sim}{\phi}\right]-\frac{1}{2} \underset{\sim}{\sim} \bar{\omega}_{2} r \\
\zeta & =\frac{1}{E}\left(g_{3}^{0} \frac{2 x_{3}}{t}+\sigma_{33}^{1} s\right)-\frac{\nu}{E} \operatorname{tr}\left({\underset{\approx}{\sigma}}^{1}\right) \frac{x_{3}}{t}-\bar{\omega}_{2} \frac{12 x_{3}}{t^{2}} \\
& =\frac{12 x_{3}}{t^{2}}\left(\omega_{2}-\bar{\omega}_{2}\right)-\frac{1}{E}\left[\frac{10}{7} \frac{x_{3}}{t}-s\left(x_{3}\right)\right] \sigma_{33}^{1} .
\end{aligned}
$$

Thus

$$
\begin{aligned}
\left\|\underline{\underline{\underline{\underline{A}}}} \underline{\underline{\sigma}}^{\mathrm{b}}-\underline{\underline{\varepsilon}}\left(\underline{\bar{u}}^{\mathrm{b}}\right)\right\|_{0, P_{t}}^{2} & \leq C t\left(\left\|{\underset{\sim}{g}}^{1}\right\|_{0}^{2}+\left\|\sigma_{33}^{1}\right\|_{0}^{2}+\|\underset{\sim}{\nabla} \omega-\underset{\sim}{\phi}\|_{0}^{2}+\left\|\underset{\sim}{\nabla} \bar{\omega}_{2}\right\|_{0}^{2}+t^{-2}\left\|\bar{\omega}_{2}-\omega_{2}\right\|_{0}^{2}\right) .
\end{aligned}
$$

If we define $\bar{\omega}_{2}$ by

$$
-t^{2} \Delta \bar{\omega}_{2}+\bar{\omega}_{2}=\omega_{2} \text { in } \Omega, \quad \bar{\omega}_{2}=0 \text { on } \partial \Omega,
$$

we obtain from Lemma 6 that

$$
\left\|\underset{\sim}{\nabla} \bar{\omega}_{2}\right\|_{0}^{2}+t^{-2}\left\|\bar{\omega}_{2}-\omega_{2}\right\|_{0}^{2} \leq C t^{-1}\left\|\omega_{2}\right\|_{1}^{2} .
$$

Substituting this estimate in (30) and invoking Lemma 5 we get

$$
\begin{aligned}
\| \underline{\underline{\underline{\underline{A}}}} & \underline{\underline{\sigma}}^{\mathrm{b}}-\underline{\underline{\varepsilon}}\left(\underline{\underline{u}}^{\mathrm{b}}\right) \|_{0, P_{t}} \leq C\left(t^{-1}\left\|g_{3}^{0}\right\|_{-1}+t^{1 / 2}\left\|g_{3}^{0}\right\|_{0}+t\left\|g_{3}^{0}\right\|_{1}+t^{-1}\left\|f_{3}^{0}\right\|_{-1}\right. \\
& \left.+t^{1 / 2}\left\|f_{3}^{2}\right\|_{0}+t\left\|f_{3}^{2}\right\|_{1}+\|\underset{\sim}{g}\|_{0}+t^{3 / 2}\|\operatorname{div} \underset{\sim}{g}\|_{0}+t^{2}\left\|\operatorname{div}{\underset{\sim}{g}}^{1}\right\|_{1}+\left\|{\underset{\sim}{f}}^{1}\right\|_{0}\right) .
\end{aligned}
$$


REMARK. Again, the use of $\underline{\bar{u}}^{\mathrm{b}}$ instead of $\underline{u}^{\mathrm{b}}$ (which is necessary to satisfy the boundary conditions) results in the loss of half a power of $t$. Indeed one can check that

$$
\begin{aligned}
\left\|\underline{\underline{\underline{\underline{A}}}} \underline{\underline{\sigma}}^{\mathrm{b}}-\underline{\underline{\varepsilon}}\left(\underline{\underline{u}}^{\mathrm{b}}\right)\right\|_{0, P_{t}} \leq C t^{1 / 2}\left(t^{-1}\left\|g_{3}^{0}\right\|_{-1}+\left\|g_{3}^{0}\right\|_{0}+t\left\|g_{3}^{0}\right\|_{1}+t^{-1}\left\|f_{3}^{0}\right\|_{-1}\right. \\
\left.+\left\|f_{3}^{2}\right\|_{0}+t\left\|f_{3}^{2}\right\|_{1}+\|\underset{\sim}{g}\|_{0}+t\left\|\operatorname{div} \underset{\sim}{g^{1}}\right\|_{0}+t^{2}\left\|\operatorname{div} \underset{\sim}{g^{1}}\right\|_{1}+\|\underset{\sim}{f}\|_{0}\right)
\end{aligned}
$$

We also obtain

$$
\begin{aligned}
\left\|\underline{\underline{\varepsilon}}\left(\underline{\bar{u}}^{\mathrm{b}}-\underline{u}^{\mathrm{b}}\right)\right\|_{0, P_{t}} \leq C t^{1 / 2}\left[\left\|\underset{\sim}{\nabla}\left(\bar{\omega}_{2}-\omega_{2}\right)\right\|_{0}+t^{-1}\left\|\bar{\omega}_{2}-\omega_{2}\right\|_{0}\right] \leq\left\|\omega_{2}\right\|_{1} \\
\leq C\left(t^{-1}\left\|g_{3}^{0}\right\|_{-1}+t\left\|g_{3}^{0}\right\|_{1}+t^{-1}\left\|f_{3}^{0}\right\|_{-1}+t\left\|f_{3}^{2}\right\|_{1}\right. \\
\left.+\|\underset{\sim}{g}\|_{0}+t^{2}\left\|\operatorname{div} \underset{\sim}{g^{1}}\right\|_{1}+\left\|\underset{\sim}{f^{1}}\right\|_{0}\right) .
\end{aligned}
$$

Combining the bounds (28) and (31) and invoking the two energies principle, we obtain

$$
\begin{aligned}
\left\|\underline{\underline{\varepsilon}}\left(\underline{u}^{*}-\underline{\bar{u}}\right)\right\|_{0, P_{t}}+\left\|\underline{\underline{\sigma}}^{*}-\underline{\underline{\sigma}}\right\|_{0, P_{t}} & \leq C\left[N\left({\underset{\sim}{g}}^{0}\right)+N\left(\underset{\sim}{f^{0}}\right)+t N\left(g_{3}^{1}\right)+t^{1 / 2} N\left(f_{3}^{1}\right)\right. \\
& \left.+t^{-1} N\left(g_{3}^{0}\right)+t^{-1} N\left(f_{3}^{0}, f_{3}^{2}\right)+N\left({\underset{\sim}{g}}^{1}\right)+N\left({\underset{\sim}{f}}^{1}\right)\right]
\end{aligned}
$$

which is the estimate of Theorem 1 but with $\underline{u}$ replaced by $\underline{\bar{u}}$. Invoking (29) and (32) concludes the proof of the theorem.

\section{Further Discussion}

The application of the two energies principle to justify plate theories was initiated in the pioneering work of Morgenstern [8], where it was used to prove convergence of the biharmonic model of plate bending to the three-dimensional solution when the thickness tends to zero. The biharmonic model is not derived in that work and the expressions of the approximate stress and displacement in terms of the biharmonic solution are chosen in an ad hoc fashion, as needed for the convergence proof. Although the rate of convergence is not considered, the residual error is not unlike that treated here and one can obtain the same $O\left(t^{1 / 2}\right)$ rate in relative energy norm.

Besides the variational approach used here, there are two other main approaches to the derivation of plate models, each with many variations. We do not attempt to review the vast literature. Surveys may be found in [6] and [11]. The most classical approach starts with a priori assumptions on the form of some components of the solution, or on constraints that the solution must satisfy. These are justified on physical or mechanical grounds as an attempt to capture the fact that the plate is thin. This approach does not seem to lend itself naturally to an error analysis. The second main approach is to use asymptotic analysis in which the plate thickness is viewed as a small parameter in the three-dimensional boundary-value problem and, with the help of assumptions on the data and scaling of the independent and/or dependent variables, a formal or rigorous passage to the limit of zero thickness is made. A very systematic treatment based on asymptotic analysis, can be found in the book [6] of Ciarlet. In the approach of Ciarlet and his coworkers, asymptotic analysis is not only used to derive a plate model, but also to rigorously justify convergence of the (appropriately scaled) 3-D solution to the (appropriately scaled) 
plate model solution. While no rate of convergence is found in that work, an $O\left(t^{1 / 2}\right)$ estimate is proved in [7]. It is worth noting that the approach presented here, in contrast to the asymptotic approach, requires no assumptions on the scaling of the loads, in order to obtain the convergence result.

The asymptotic approach, as present in [6], leads to essentially the same model of plate stretching as was obtained here by the $\mathrm{HR}_{1}(1)$ method, namely the generalized plane stress problem in which the only loads are due to the even part of the in-plane surface load and the average over the plate thickness of the in-plane volume load. Recall that that equation, unlike the variant we obtained via $\operatorname{HR}_{4}^{\prime}(1)$, ignores the effect of the odd part of the transverse loads on stretching.

For plate bending, the asymptotic approach leads to the Kirchhoff-Love or biharmonic plate equation, rather than to the Reissner-Mindlin equations. (It is easy to see that the biharmonic model is, after appropriate scaling, the zero thickness limit of the Reissner-Mindlin model.) To the best of our knowledge, there is no way to obtain Reissner-Mindlin type models of plate bending from the asymptotic approach. On the other hand, the variational approach presented here gives rise to many different methods, but the simplest model it yields is Reissner-Mindlin. We know of no way to obtain the biharmonic model from this approach without either making a priori mechanical assumptions (e.g., by enforcing the Kirchhoff hypothesis on the trial space), or by passing to a limit of zero thickness after obtaining the twodimensional model. We also remark again that the minimum energy approaches $\left(\mathrm{HR}_{2}(p)\right.$ and $\left.\mathrm{HR}_{3}(p)\right)$ do not yield either the plane stress system, nor the biharmonic equation, nor the Reissner-Mindlin system. It is sometimes suggested to obtain the Reissner-Mindlin system from the (divergent) $\mathrm{HR}_{2}(1)$ approach, by changing the compliance tensor in an ad hoc fashion. However it is difficult to justify this procedure except that it yields a model that is known from other derivations. In our opinion, a preferable procedure is to use the $\mathrm{HR}_{1}(1)$ model and eliminate the stress field in order to obtain, in a rational manner, a modification of the displacement energy form to minimize.

A striking feature of the error analysis is the slow rate of convergence obtained, namely $O\left(t^{1 / 2}\right)$. Recall that in our analysis of the $\mathrm{HR}_{4}^{\prime}(1)$ model, this rate arises as a consequence of the failure of the model to match the boundary conditions on the lateral boundary. Even for the formally higher-order methods, $\operatorname{HR}_{4}^{\prime}(p), p>1$, the same difficulty will arise, and so these methods are not found to be of truly higher order. (This statement holds for most realistic plate boundary-value problems. For periodic plates, or plates subject to very special conditions on the lateral boundary, higher-order convergence is possible.) One might wonder whether the $O\left(t^{1 / 2}\right)$ rate is realistic, or only a pessimistic consequence of the method of proof. Although we know of no definite results for the model treated here, it appears to be real. Combining the two energies approach with a more accurate choice of the boundary corrector $\left(\bar{\omega}_{2}-\omega_{2}\right.$ in our notation), and an asymptotic analysis of its behavior, Chen was able to prove that the $O\left(t^{1 / 2}\right)$ rate is sharp for the biharmonic plate bending model in some situations [5]. He also investigated the question of whether higherorder error estimates hold for the restriction of the error to a subdomain which stays away from the lateral boundary, and, again for the biharmonic, answered this affirmatively for some boundary-value problems. 


\section{Appendix: Derivation of the $\operatorname{HR}_{4}^{\prime}(1)$ model}

In view of the definition of the spaces $\sum_{g}^{*}$ and $\underline{V}^{*}$ in $\S 3$, the $\operatorname{HR}_{4}^{\prime}(1)$ solution may be written in the form (12), (13) for some functions $\underset{\sim}{\eta}, \rho, \underset{\sim}{\phi}, \omega, \omega_{2}, \underset{\approx}{\sigma^{0}}, \sigma_{33}^{0}, \underset{\approx}{\sigma^{1}}$, $\sigma^{0}$, and $\sigma_{33}^{1}$ of $\underset{\sim}{x}$. We now verify that these functions satisfy (and so are determined by) equations (14)-(22).

To assist in the computations below we record the following integrals, all taken over the interval $(-t / 2, t / 2)$ :

$$
\begin{gathered}
\int q(z) d z=t, \quad \int z q(z) d z=0, \quad \int q(z)^{2} d z=6 t / 5, \quad \int z q^{\prime}(z) d z=-t \\
\int r(z) d z=t / 5, \quad \int s^{\prime}(z) d z=0, \quad \int s(z) z d z=t^{2} / 12, \quad \int s(z)^{2} d z=5 t / 42 \\
\int q(z) r(z) d z=0, \quad \int s^{\prime}(z) r(z) d z=-1, \quad \int z^{2} d z=t^{3} / 12 .
\end{gathered}
$$

Turning first to the verification of the stretching problem, we note that

$$
\begin{aligned}
& \underline{\underline{\underline{\underline{A}}}} \underline{\underline{\sigma}}^{\mathrm{s}}(\underline{x})
\end{aligned}
$$

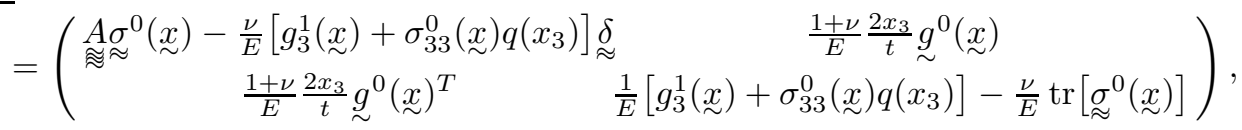

and

$$
\operatorname{div} \underline{\underline{\sigma}}^{\mathrm{s}}(\underline{x})=\left(\begin{array}{c}
\operatorname{div}_{\sim}^{\operatorname{div}} \underset{\widetilde{\sigma}}{\sigma^{0}}(\underset{\sim}{x})+\frac{2}{t} \underline{\sim}^{0}(\underset{\sim}{x}) \\
\underline{2 x_{3}} \operatorname{div} \underset{\sim}{\operatorname{gin}^{0}}(\underset{\sim}{x})+\sigma_{33}^{0}(\underset{\sim}{x}) q^{\prime}\left(x_{3}\right)
\end{array}\right) .
$$

We shall derive the equations for the stretching portion of the solution, (14)(17), by taking a sequence of test functions in (9) and (10). First, let $v \in L^{2}(\Omega)$ be arbitrary and take $\underline{v}(\underline{x})=\left(0, v(\underset{\sim}{x}) x_{3}\right)^{T}$ in $(10)$. This gives the expression $(17 \mathrm{~b})$ for $\sigma_{33}^{0}$. Next take $\underline{v}=(\underset{\sim}{v}, 0)^{T}$ where $\underset{\sim}{v} \in \underset{\sim}{L}(\Omega)$ is arbitrary, obtaining

$$
-t \operatorname{div}_{\sim} \underset{\sim}{\sigma^{0}}={\underset{\sim}{l}}_{1}
$$

Third, let $\underset{\sim}{\tau} \in \underset{\sim}{H}(\operatorname{div}, \Omega)$ be arbitrary and choose the test function

$$
\underline{\underline{\tau}}=\left(\begin{array}{cc}
\tau & 0 \\
\widetilde{0} & 0
\end{array}\right)
$$

in (9). Noting that $\underline{\operatorname{div}} \underline{\underline{\tau}}=\left(\operatorname{div}_{\sim} \underset{\sim}{\tau}, 0\right)^{T}$, we get

$$
\left.\int_{\Omega}\left[\underset{\approx}{A \sigma^{0}}: \underset{\approx}{\tau}-\frac{\nu}{E}\left(\sigma_{33}^{0}+g_{3}^{1}\right) \operatorname{tr}(\underset{\approx}{\tau})+\underset{\sim}{\eta} \cdot \underset{\sim}{\operatorname{div}} \underset{\approx}{\tau}\right] d \underset{\sim}{x}=0 \quad \text { for all } \underset{\approx}{\underset{\approx}{\sim}} \underset{\sim}{\operatorname{div}}, \Omega\right) .
$$

This implies the boundary condition (15) and that

$$
\underset{\approx \approx}{A \sigma^{0}}=\underset{\approx}{\varepsilon}(\underset{\sim}{\eta})+\frac{\nu}{E}\left(\sigma_{33}^{0}+g_{3}^{1}\right) \underset{\approx}{\delta}=\underset{\approx}{\varepsilon}(\underset{\sim}{\eta})+\frac{\nu}{E} l_{2} \underset{\approx}{\delta}
$$

where we have used (17b) and (16) to obtain the last equality. This immediately gives (17a) and, combining with (33), we obtain the differential equation (14). Thus 
we have verified that $\underset{\sim}{\eta}$ is determined by (14) and (15), $\sigma_{\sim}^{0}$ is determined by (17a), and $\sigma_{33}^{0}$ is determined by $(17 \mathrm{~b})$. To obtain $(17 \mathrm{c})$, we choose

$$
\underline{\underline{\tau}}=\left(\begin{array}{cc}
0 & 0 \\
0 & \tau q\left(x_{3}\right)
\end{array}\right)
$$

in (9) with $\tau \in L^{2}(\Omega)$ arbitrary. Thus we have verified the stretching solution for $\operatorname{HR}_{4}^{\prime}(1)$.

For the bending problem, we note that

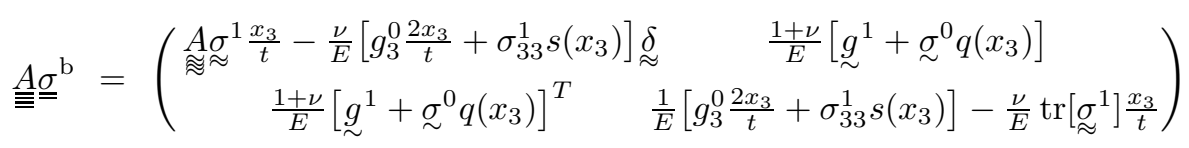

and

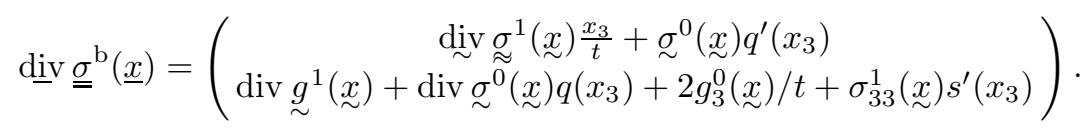

Let $v \in L^{2}(\Omega)$ be arbitrary and take $\left.\underline{v}(\underline{x})=(0, v \underset{\sim}{x})\right)^{T}$ in $(10)$. This yields the equation

$$
-t \operatorname{div} \underset{\sim}{\sigma^{0}}=t \operatorname{div} \underset{\sim}{g^{1}}+2 g_{3}^{0}+f_{3}^{0} .
$$

Next, take $\underline{v}(\underline{x})=\left(0, v(\underset{\sim}{x}) r\left(x_{3}\right)\right)^{T}$ where again $v \in L^{2}(\Omega)$ is arbitrary, to obtain (22c).

Third, let $\underline{v}(\underline{x})=\left(\underset{\sim}{\psi}(\underset{\sim}{x}) x_{3}, 0\right)^{T}$ with $\underset{\sim}{\psi} \in{\underset{\sim}{L}}^{2}(\Omega)$ arbitrary, to get

$$
-\frac{t^{2}}{12} \underset{\sim}{\operatorname{div}} \underset{\approx}{\sigma^{1}}+t \underset{\sim}{\sigma^{0}}=t \underset{\sim}{f^{1}}
$$

Next we take various choices of $\underset{\underline{\tau}}{\text { in }}(9)$. First let $\underset{\sim}{\tau} \in \underset{\sim}{H}(\underset{\sim}{\operatorname{div}}, \Omega)$ be arbitrary and take

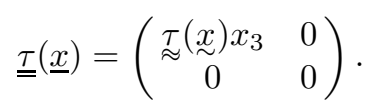

Noting that $\underline{\operatorname{div}} \underline{\underline{\tau}}=\left(\underset{\sim}{\operatorname{div}} \underset{\sim}{\tau} x_{3}, 0\right)^{T}$, we get, for all $\underset{\sim}{\tau} \in \underset{\sim}{H}(\operatorname{div}, \Omega)$, that

$$
\int_{\Omega}\left[\frac{t^{2}}{12} \underset{\approx}{\approx \sigma^{1}}: \tau-\frac{t^{2}}{6} \frac{\nu}{E} g_{3}^{0} \operatorname{tr}(\underset{\approx}{\tau})-\frac{\nu}{E} \frac{t^{2}}{12} \sigma_{33}^{1} \operatorname{tr}(\underset{\approx}{\tau})-\frac{t^{3}}{12} \underset{\sim}{\phi} \cdot \underset{\sim}{\operatorname{div}} \underset{\approx}{\tau}\right] d \underset{\sim}{x}=0 .
$$

This implies that

and that

$$
\underset{\sim}{\phi}=0 \text { on } \partial \Omega
$$

or

$$
\underset{\approx}{A \sigma^{1}}{ }^{1}=-t \underset{\approx}{\varepsilon}(\underset{\sim}{\phi})+\frac{\nu}{E}\left(\sigma_{33}^{1}+2 g_{3}^{0}\right) \underset{\approx}{\delta},
$$

$$
\begin{aligned}
& {\underset{\sim}{\sigma^{1}}}^{1}=-t \underset{\approx}{A_{\tilde{\approx}}^{-1}} \underset{\sim}{\varepsilon}(\phi)+\frac{\nu}{1-\nu}\left(\sigma_{33}^{1}+2 g_{3}^{0}\right) \underset{\sim}{\delta}
\end{aligned}
$$

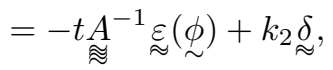

where we have invoked (22c) at the last step. Thus (22a) is satisfied. 
Next set

$$
\underline{\underline{\tau}}(\underline{x})=\left(\begin{array}{cc}
0 & \underset{\tau}{\tau}(\underset{\sim}{x}) q\left(x_{3}\right) \\
\underset{\sim}{\tau}(x)^{T} q\left(x_{3}\right) & 0
\end{array}\right)
$$

with $\underset{\sim}{\tau} \in \underset{\sim}{H}(\operatorname{div}, \Omega)$. We find that

$$
\int_{\Omega}\left\{t\left[\frac{2(1+\nu)}{E}\left({\underset{\sim}{g}}^{1}+\frac{6}{5} \sigma^{0}\right)+\underset{\sim}{\phi}\right] \cdot \underset{\sim}{\tau}+t \omega \operatorname{div} \underset{\sim}{\tau}\right\} d \underset{\sim}{x}=0
$$

for all such $\underset{\sim}{\tau}$. We infer that

and (22b) holds.

$$
\omega=0 \text { on } \partial \Omega,
$$

Substituting (22b) into (34) leads to the equation (19). Similarly, inserting (36) and (22b) into (35) we obtain (18). We have thus verified (18)-(20) and the first three equations of (22). To obtain the final equation we choose the test function

$$
\underline{\underline{\tau}}(\underline{x})=\left(\begin{array}{cc}
0 & \underset{\sim}{0}\left(x_{3}\right) \\
0 & \tau(\underset{\sim}{x})
\end{array}\right)
$$

in (9).

\section{References}

[1] S. M. Alessandrini, Some two-dimensional plate models: Derivation, asymptotic properties, and numerical approximation, Ph.D. thesis, Rutgers Univ., New Jersey, 1991.

[2] D. N. Arnold and R. S. Falk, A uniformly accurate finite element method for the ReissnerMindlin plate model, SIAM J. Numer. Anal. 26 (1989), 1276-1290.

[3] Asymptotic analysis of the boundary layer for the Reissner-Mindlin plate model, SIAM J. Math. Anal. 27 (1996), 486-514.

[4] I. Babuška and L. Li, The problem of plate modeling: Theoretical and computational results, Comput. Methods Appl. Mech. Engrg. 100 (1992), 249-273.

[5] C. Chen, Asymptotic convergence rates for the Kirchhoff plate model, Ph.D. thesis, Univ. Park, Pennsylvania, 1995.

[6] P. G. Ciarlet, Plates and junctions in elastic multi-structures, an asymptotic analysis, Masson, Paris, 1990.

[7] P. Destuynder, Sur une justification des modèles de plaques et de coques par les méthodes asymptotiques, Ph.D. thesis, Univ. P. et M. Curie, Paris, 1980.

[13] K. H. Lo, R. M. Christensen, and E. M. Wu, A high-order theory of plate deformations, J. Appl. Mech. 46 (1977), 663-676.

[8] D. Morgenstern, Herleitung der Plattentheorie aus der dreidimensionalen Elastizitätstheorie, Arch. Rational Mech. Anal. 4 (1959), 145-152.

[9] W. Prager and J. L. Synge, Approximations in elasticity based on the concept of the function space, Quart. Appl. Math. 5 (1947), 241-269.

[10] E. Reissner, On bending of elastic plates, Quart. Appl. Math. 5 (1947), 55-68.

[11] _ Reflections on the theory of elastic plates, Appl. Mech. Rev. 38 (1985), 1453-1464.

[12] C. Schwab, A-posteriori modeling error estimation for hierarchic plate models, Numer. Math. 74 (1996), 221-259.

Lockheed Martin Corporation, Bullding 137-123, 199 Borton Landing Road, MOORESTOWn, NJ 08057, USA

E-mail address: stephen.m.alessandrini@lmco.com

Department of Mathematics, The Pennsylvania State University, University PARK, PA 16802, USA

E-mail address: dna@math.psu.edu 
DERIVATION AND JUSTIFICATION OF PLATE MODELS BY VARIATIONAL METHODS 21

Department of Mathematics, Rutgers University, Piscatway, NJ 08854, USA

E-mail address: falk@math.rutgers.edu

Department of Mathematics, The Pennsylvania State University, University PARK, PA 16802, USA

E-mail address: alm@math.psu.edu 